\title{
Alianças Estratégicas: uma análise bibliométrica da produção científica entre 1989 e 2008
}

\author{
Márcio Roberto Moran ${ }^{1}$ \\ Fernando Ferreira de Araujo Souza ${ }^{2}$ \\ João Maurício Gama Boaventura ${ }^{3}$ \\ Bernadete de Lourdes Marinho ${ }^{4}$ \\ Adalberto Américo Fischmann ${ }^{5}$
}

\section{Resumo}

Considera-se pertinente a organização de dados sobre a produção científica de um período, uma vez que tal movimento contribui para a continuidade das investigações e a evolução de determinada área do conhecimento. O propósito principal deste estudo, portanto, é analisar quantitativamente o que se produziu no campo das alianças estratégicas entre 1989 e 2008. Apoiando-se em técnicas bibliométricas e na pesquisa bibliográfica, foi possível encontrar 66 expressões que conduzem pesquisadores aos mais variados conteúdos sobre o tema em questão. Além disso, a partir de consultas às bases ISI Web of Knowledge e Scopus constituíram-se duas amostras, respectivamente, com 2.747 e 2.813 pesquisas publicadas, que permitiram a identificação dos autores, artigos e periódicos mais referenciados por estudiosos em todo o mundo. Este trabalho, então, torna-se um importante aliado no desenvolvimento de novas ideias, conceitos e perspectivas de abordagens sobre alianças estratégicas.

Palavras-chave: Alianças estratégicas. Análise bibliométrica. Parcerias estratégicas.

\footnotetext{
${ }^{1}$ Economista, Mestrando em Administração. Faculdade de Economia, Administração e Contabilidade. Universidade de São Paulo. Endereço: Rua Inhatium, 245 ap. 22 - Alto de Pinheiro -São Paulo/SP - Brasil. 05468-160.E-mail: marcio.moran@usp.br.

${ }^{2}$ Escola Engenheiro, Mestrando do Núcleo de Real Estate. Escola Politécnica. Universidade de São Paulo. Endereço: Av. Professor Almeida Prado, travessa 2, n. 83. Ed. de Engenharia Civil. Bairro Cidade Universitária, São Paulo, SP, Brasil. CEP: 05508-900. E-mail: ffasouza@gmail.com.

${ }^{3}$ Doutor em Administração. Fundação Instituto de Administração - FIA. Endereço: Rua Itapaiuna, 1800 Apto 221-S São Paulo/SP - Brasil. CEP 05707-001.E-mail: jm@boaventura.adm.br.

${ }^{4}$ Professora doutora da Faculdade de Economia, Administração e Contabilidade. Universidade de São Paulo. Endereço: Av. Professor Luciano Gualberto, 908 Sala E-105 - São Paulo/SP - Brasil. CEP 05508-900. E-mail: marinhoy@usp.br.

${ }^{5}$ Professor Titular. Instituição à qual está vinculado: Faculdade de Economia, Administração e Contabilidade. Universidade de São Paulo. Endereço: Av. Professor Luciano Gualberto, 908 - São Paulo/SP - Brasil. CEP 05508-900. E-mail: aafischm@usp.br.

Artigo recebido em: 15/12/2009. Aceito em: 1\%07/2010. Membro do Corpo Editorial Científico responsável pelo processo editorial: Thomas G. Brashear.
} 


\section{Introdução e Justificativa}

A crescente integração do mercado mundial nas últimas décadas - a globalização - tem exigido das organizações novas formas de atuação em muitas indústrias. As alianças estratégicas, especificamente, têm sido utilizadas por executivos com o intuito de aumentar a competitividade das entidades as quais representam. A variedade e o número de iniciativas desse tipo tornam o referido tema um tópico relevante quando o assunto é gestão. (YOSHINO; RAGAN, 1995).

O avanço científico nesse campo de estudo, portanto, deriva, fundamentalmente, do conhecimento disponível sobre ele, que é fruto de investigações e interações entre pesquisadores ao longo do tempo. Conhecer a evolução dos conceitos, os principais estudiosos e a produção acadêmica de maior relevância pode colaborar significativamente para a melhor compreensão da teoria existente e para a identificação dos problemas que ainda não foram solucionados.

Desenvolvido a partir dessa visão, o objetivo principal deste trabalho é analisar a produção científica sobre alianças estratégicas entre organizações de maneira quantitativa. Os objetivos específicos, por sua vez, são: (1) identificar as expressões-chave que levam pesquisadores a encontrar materiais bibliográficos sobre alianças estratégicas; (2) identificar os autores e artigos mais relevantes no campo das alianças estratégicas entre organizações; e (3) identificar os periódicos mais referenciados no assunto entre 1989 e 2008. Optou-se pelo período indicado, uma vez que a análise dos últimos 20 anos é suficiente para que se encontrem conceitos consolidados historicamente e tendências de linhas de pesquisa.

A fim de que os objetivos indicados possam ser alcançados, duas ferramentas metodológicas foram utilizadas, a bibliometria e a pesquisa bibliográfica. Cabe destacar que uma consulta preliminar à base ISI (2009) não localizou trabalhos bibliométricos com foco em alianças estratégicas. Uma busca ampliada na área de estratégia e gestão, porém, permitiu a identificação de dois estudos desenvolvidos com o referido método - RamosRodriguez e Ruiz-Navarro (2004) e Pilkington e Meredith (2009).

Este artigo, então, divide-se em seis partes: a primeira introduz o leitor ao objeto de pesquisa; a segunda aponta as definições operacionais do trabalho; a terceira apresenta uma breve revisão bibliográfica sobre as formas de conceituação do termo alianças estratégicas; a quarta descreve o método 
e as técnicas aplicadas ao longo do estudo; enquanto a quinta seção demonstra os resultados; por fim, a sexta parte compõe-se das considerações finais, das limitações deste trabalho e das sugestões para futuras pesquisas.

\section{Definições Operacionais}

A fim de evitar diferentes interpretações sobre o que venham a ser as chamadas expressões-chave, autores e artigos mais relevantes, periódicos e alianças estratégicas entre organizações, são oferecidas as definições operacionais desses termos:

a) Expressões-chave: de acordo com Ferreira (1999), expressão é a enunciação do pensamento por meio de gestos ou palavras escritas ou faladas e chave é o que prepara, facilita, explica ou inicia. Portanto, expressões-chave, neste trabalho, são as formas de exprimir e acessar a significação do termo alianças estratégicas mais facilmente.

b) Autores: segundo Ferreira (1999), são criadores de obra artística, literária ou científica. Neste estudo, considera-se apenas os escritores de obras científicas, conforme notado nas bases de dados consideradas.

c) Artigos: "são trabalhos técnicos, científicos ou culturais, escritos por um ou mais autores, que seguem as normas editoriais do periódico a que se destinam." (MARTINS; THEÓPHILO, 2007, p. 213). Nesse caso, são considerados somente os trabalhos científicos, conforme indicam as bases de dados escolhidas.

d) Relevantes: que se sobressaem ou ressaltam; de grande valor, convenientes ou interessantes; importantes; aqueles que importam ou são necessários. (FERREIRA, 1999). Julgam-se relevantes os 20 autores, artigos e periódicos mais citados, que versam sobre alianças estratégicas e que foram encontrados nas bases de dados ISI (2009) e Scopus (2009).

e) Periódicos: são publicações editadas em fascículos ou partes, a intervalos regulares ou não, por tempo indeterminado, nas quais co- 
laboram diversas pessoas, sob uma direção constituída. Pode tratar de vários assuntos em uma ou mais áreas do conhecimento. (MARTINS; THEÓPHILO, 2007). Para esta pesquisa, servem apenas os periódicos científicos, ou seja, que exigem rigor metodológico dos trabalhos submetidos para publicação e que constam nas bases de dados investigadas.

f) Alianças estratégicas: são acordos de cooperação entre firmas com vistas a atingir os objetivos estratégicos dos parceiros. (DAS; TENG, 1998).

\section{Revisão Bibliográfica}

\subsection{Alianças Estratégicas: definições e formas de conceituação}

Sejam quais forem as perspectivas de abordagem e os métodos utilizados nas pesquisas científicas sobre alianças estratégicas, todas elas obrigatoriamente, ainda que não explicitamente, carregam consigo uma definição do termo destacado. A revisão de vários textos sobre o assunto permite que se identifique não só as diferenças de conceitos presentes na literatura, mas os caminhos adotados pelos pesquisadores para chegarem a uma definição.

Das e Teng (1998), conforme enunciado no tópico anterior, conceituam as alianças estratégicas como sendo acordos de cooperação entre firmas por meio dos quais os parceiros visam atingir objetivos estratégicos previamente definidos. Gulati (1998), por sua vez, afirma que as alianças são acordos voluntários entre organizações que envolvem troca, compartilhamento ou codesenvolvimento de produtos, tecnologias ou serviços.

Na mesma linha de formulação conceitual, encontram-se os trabalhos de Mohr e Spekman (1994) e de Robinson (2008), pois, segundo os autores, alianças estratégicas são relações vantajosas entre empresas independentes que têm objetivos compatíveis, perseguem benefícios mútuos e apresentam elevado nível de dependência. Baseando-se nessa ideia, Robinson (2008) adiciona a visão de longo prazo e a partilha de custos como características das alianças. 
Outros autores, porém, recorrem a caminhos distintos para conceituar o termo em questão. Lorange e Roos (1993), por exemplo, apoiam-se numa escala contínua de integração entre organizações, que tem o mercado - ambiente marcado pela livre troca de bens, ativos e serviços - num extremo, e a hierarquia - entendida como nível de controle sobre determinado negócio no outro. As fusões e aquisições são consideradas por eles casos extremos de hierarquização e dependência mútua, enquanto as cooperações informais estão mais próximas das relações de mercado e se caracterizam por um alto grau de independência.

Lorange e Roos (1993, p. 3) escreveram:

One theorical way to define strategic alliances is to look at the continuos scale between, on the one hand, transactions on a free market ("market") and, on the other, total internalization ("hierarchy").

Baseando-se também num "contínuo de relacionamento", cujos extremos são as relações transacionais e as unificações, Ribeiro e Silva Junior (2001) apresentam uma definição distinta da oferecida por Lorange e Roos (1993). Ribeiro e Silva Junior (2001, p. 91) afirmam que uma aliança estratégica ocorre apenas "a partir do momento em que as empresas tomam a forma de um empreendimento completamente novo [...]". Os autores adicionam a esse enunciado alguns outros aspectos: as alianças devem mover cada participante em direção a um objetivo estratégico de longo prazo, pressupõem compartilhamento de objetivos, comprometimento de recursos de capital e recursos administrativos dos envolvidos, e mantêm independentes as duas ou mais organizações após a constituição da parceria.

Além das definições e formas de conceituação descritas até o momento, outro caminho encontrado na literatura é a negativa. Powell, Koput e SmithDoerr (1996), por exemplo, ao analisarem a importância das capacidades internas e externas de aprendizado de empresas em alianças no setor de biotecnologia, apontam dois aspectos que contribuem para o entendimento do referido tema: (1) colaborações interorganizacionais não são simplesmente um meio para compensar a falta de competências internas e (2) nem devem ser vistas como uma série de transações discretas.

Nota-se, portanto, que há uma série de correntes de pensamento sobre o que venham a ser as alianças estratégicas. Por essa razão, este trabalho 
considera todas as visões presentes nos estudos sobre o assunto, ainda que tenha sido cunhada uma definição operacional no tópico anterior. A última seção, ao tratar das alternativas de futuras pesquisas, reforça a importância de manter o universo desta investigação abrangente.

\section{Metodologia}

\subsection{Bibliometria: o método}

Embora o desenvolvimento deste estudo não seja possível sem uma cuidadosa pesquisa bibliográfica, faz-se necessário destacar a importância da bibliometria para a consecução dos objetivos apontados na introdução.

Lancaster (1977) e Sousa (1989), citados por Pilkington e Meredith (2009), definem bibliometria como sendo, respectivamente, (1) o emprego de padrões de escrita, publicações e de literatura pela aplicação de diversas análises estatísticas e (2) a técnica de investigação que tem por fim a análise do tamanho, crescimento e distribuição da bibliografia num determinado campo do conhecimento.

Guedes e Borschiver (2005), por sua vez, enfocam o auxílio à tomada de decisões, organização e sistematização de informações ao discorrem sobre o referido método. Para esses autores, trata-se de um instrumento quantitativo que permite minimizar a subjetividade das análises. Três são as principais leis bibliométricas descritas por eles e que tem relação direta com este trabalho: de Zipf, de Lotka e de Bradford.

Enquanto a lei de Zipf observa a frequência de ocorrência de palavras em um dado texto com o objetivo de propor indexações, a lei de Lotka trata da produtividade de autores. Esta última fundamenta-se na premissa de que alguns pesquisadores publicam muito e que muitos acadêmicos publicam pouco. A terceira lei, chamada de lei de Bradford, permite estimar o grau de relevância de periódicos numa determinada área do conhecimento. Nesse caso, uma vez que os primeiros artigos sobre um novo assunto são escritos e publicados por periódicos apropriados, tais veículos atraem mais artigos sobre o tema em questão, gerando um ciclo de retroalimentação que acelera a construção de uma imagem positiva de determinados journals numa área de conhecimento. 
Todos os três conceitos são facilmente identificados na apresentação dos resultados desta pesquisa. O primeiro deles está associado às principais expressões-chave que cercam o tema alianças estratégicas. $\mathrm{O}$ segundo aparece refletido no quadro que indica os autores com produção mais reconhecida, medida pelo número de citações e o número de artigos publicados. A terceira, por fim, pode ser vista na análise dos principais periódicos que tratam de alianças estratégicas, cuja tabela indica a credibilidade do periódico no referido tema e a frequência de publicações nos últimos 20 anos.

As leis bibliométricas, portanto, fazem uso da análise matemática e estatística de dados para investigar e quantificar a produção científica sobre determinado assunto. Isso posto, convém também destacar alguns importantes princípios do método: a citação, a cocitação e a teoria epidêmica de Goffman.

Segundo Araujo (2006), a análise de citação é uma técnica que possibilita a identificação de vários padrões na produção do conhecimento científico, tais como: autores mais citados, mais produtivos, elite de pesquisa $e$ procedência geográfica.

A análise de cocitação, de acordo com Guedes e Borschiver (2005), mede o grau de ligação entre dois ou mais artigos pelo número de documentos que os citam. A análise de cocitação, então, é importante à medida que identifica grupos de conhecimento (ou frentes de pesquisas), estabelecidos por conjuntos de autores que frequentemente se referenciam.

Por fim, a teoria epidêmica de transmissão de ideias, desenvolvida por Goffman e Newill (1967), citados por Araujo (2006), trata da dinâmica da transmissão do conhecimento em redes sociais. Segundo os pesquisadores, o conceito é análogo ao processo de transmissão de doenças infecciosas (processo epidêmico), sendo (1) a pessoa com a doença o autor de uma ideia; (2) o indivíduo susceptível a doença o leitor que recebe a ideia; e (3) o material infectante as próprias ideias contidas na literatura. Quanto melhores forem as condições externas para o desenvolvimento epidêmico (propagação da informação), maior será a propagação da doença.

Sendo assim, é possível identificar a importância da bibliometria para o avanço do conhecimento sobre as alianças estratégicas. Neste trabalho, o método contribui para a organização e identificação das expressões-chave, dos principais autores, artigos e periódicos relacionados ao tema, o que o torna um importante aliado no desenvolvimento de novas ideias, conceitos e perspectivas de abordagens. No tópico Análise dos Resultados, mostra-se 
como as leis e princípios bibliométricos ajudam na consecução dos objetivos do estudo.

\subsection{Formulação e Delineamento da Pesquisa}

Os objetivos geral e específicos deste estudo, indicados no tópico introdutório, derivam do problema de pesquisa selecionado e das questões que orientam toda a investigação.

a) Questão-básica: qual é o estado da arte do conhecimento sobre alianças estratégicas entre organizações?

b) Questão-problema: quais são as expressões-chave de pesquisa e os autores, artigos e periódicos mais relevantes sobre o tema alianças estratégicas entre organizações?

O passo seguinte à definição da pergunta-problema é a escolha das variáveis e premissas para as quais o método destacado anteriormente se aplica. Quanto à determinação da relevância dos autores e artigos, fez-se uso da técnica de análise de citação ao atribuir-se ao número de citações a condição de variável proxi. Guedes e Borschiver (2005) embasam a decisão ao afirmarem que a técnica parte da hipótese de que citação é um indicador válido de influência de uma determinada fonte. Para a determinação do grau de atividade dos autores e periódicos, por sua vez, considerou-se a variável número de artigos publicados no período 1989-2008.

A Figura 1 ilustra o caminho percorrido desde o problema até os resultados gerados neste trabalho: 


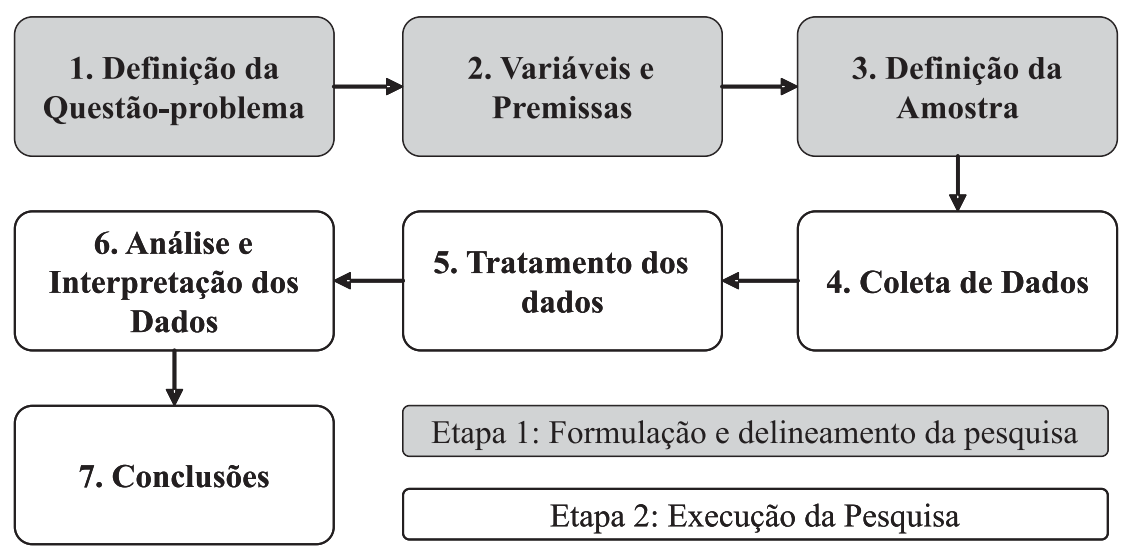

Figura 1: Processo de pesquisa

Fonte: Elaborada pelos autores

O terceiro passo na etapa de formulação e delineamento da pesquisa é a definição da amostra. Composta a partir da alternância de coleta e análise de dados e revisão bibliográfica, o subconjunto do universo de artigos sobre alianças estratégicas começou a se definir quando: (1) determinou-se a primeira base de dados - ISI (2009) - (2) decidiu-se por um único tipo de publicação - artigos acadêmicos e (3) escolheu-se quatro expressões-chave para formação de um banco de dados preliminar - strategic alliance(s) e partnership alliance(s).

Com o auxílio do EndNote Program - software que auxilia na constituição de bancos de dados bibliográficos - foi possível a organização de 2.346 elementos com o seguinte conteúdo: nomes dos autores, ano de publicação, título do artigo, periódico ou evento, tipo de publicação (artigo acadêmico ou patente), número de citações, resumo (abstract) e código de identificação do título na base. Outros dados estavam disponíveis, porém foram descartados por não contribuírem para os resultados da pesquisa.

Procedeu-se então o tratamento dos dados com o intuito de eliminar duplicidades, registros que estivessem incompletos e tipos de fontes que não correspondessem à categoria artigos acadêmicos. Vale observar que nesta etapa não foram eliminadas as publicações anteriores a 1989 e posteriores a 2008. Resultaram do esforço descrito 1.764 artigos.

Uma vez organizados os elementos pré-amostrais em planilha eletrônica, esses foram ordenados decrescentemente, por número de citações, com o intuito de se identificar os mais referenciados dentre aqueles do conjunto. 
Era prevista para o momento imediatamente seguinte uma revisão bibliográfica, portanto os autores deste estudo arbitraram um corte temporal e criaram cinco períodos distintos. Pretendia-se com tal arbitrariedade a redução da probabilidade de viés, uma vez que os artigos mais destacados concentravam-se entre 1990 e 2004.

Então, os dez artigos mais citados a cada cinco anos (1985-1989; 19901994; 1995-1999; 2000-2004; e 2005-2009) foram alvos de uma revisão em busca de palavras ou expressões que fossem sinônimos ou tivessem significados correlatos ao termo alianças estratégicas. Embora não tenha sido feita uma análise de conteúdo propriamente dita, a revisão bibliográfica conduziu os autores a 66 expressões, que uma vez usadas em substituição ao termo de referência, não alteram o sentido das ideias expostas nas pesquisas publicadas.

A fim de dirimir as dúvidas e eliminar os possíveis questionamentos, expressões relacionadas ao tema em questão e que aparecem com frequência em diversos trabalhos acadêmicos, como joint research and development, joint-ventures, mergers\&aquisitions, networks, minority equity stake, dentre outras, não integram a lista composta. Vale lembrar que muitas delas são apenas formas de caracterização de uma aliança estratégica, enquanto outras são representações de um conjunto de alianças. A Tabela 1 mostra o resultado encontrado.

Tabela 1: Sessenta e seis expressões-chave de busca

\begin{tabular}{|l|l|l|}
\hline Corporate Alliance(s) & Interorganisational Collaboration & Inter-organizational Cooperation(s) \\
\hline Corporate Partnering & Inter-organisational Collaboration(s) & Interorganizational Linkage(s) \\
\hline Interfirm Alliance(s) & Interorganisational Cooperation & Inter-organizational Linkages \\
\hline Inter-firm Alliance(s) & Interorganisational Linkages & Interorganizational Partnership(s) \\
\hline Interfirm Collaboration(s) & Inter-organisational Linkages & Inter-organizational Partnership(s) \\
\hline Inter-firm Collaboration(s) & Interorganisational Partnerships & Interorganizational Relationship(s) \\
\hline Interfirm Cooperation & Inter-organisational Relationship(s) & Inter-organizational Relationship(s) \\
\hline Inter-firm Cooperation & Interorganisational Relationships & Partner Collaboration \\
\hline Interfirm Linkage(s) & Interorganizational Alliance(s) & Partner Cooperation \\
\hline Inter-firm Linkage(s) & Inter-organizational Alliance(s) & Partners Collaboration \\
\hline Interfirm Partnership(s) & Interorganizational Collaboration(s) & Partners Cooperation \\
\hline Inter-firm Partnership(s) & Inter-organizational Collaboration(s) & Strategic Alliance(s) \\
\hline Interorganisational Alliance & Interorganizational Collaborative Relationships & Strategic Partnering \\
\hline Inter-organisational Alliances & Interorganizational Cooperation(s) & Strategic Partnership(s) \\
\hline
\end{tabular}

Fonte: Elaborada pelos autores 
Apesar desta seção tratar, exclusivamente, da metodologia empregada neste estudo, e não dos resultados, é importante mencionar que parte da resposta à questão-problema ou, simplesmente, parte dos objetivos anunciados na introdução acaba de ser alcançada. Em outras palavras, a Tabela 1 identifica as expressões-chave que permitem os pesquisadores encontrar materiais bibliográficos sobre alianças estratégicas mais precisamente.

A composição da amostra, então, foi concluída de acordo com o que segue. Quanto à determinação da base de dados, optou-se por utilizar as plataformas mais populares para pesquisas acadêmicas - ISI Web of Knowledge e Scopus (ARCHAMBAULT et al.,2009). A escolha de duas plataformas pretende a validação qualitativa dos resultados, uma vez que os produtos de ambas são analisados separadamente. Segundo Martins e Theóphilo (2007), a triangulação de fontes de dados distintas aumenta o grau de confiabilidade do estudo.

Quanto à determinação do tipo de publicação - artigos publicados em periódicos acadêmicos, anais de congressos, livros, patentes, etc. - optou-se por considerar apenas os artigos publicados em periódicos acadêmicos e congressos. Entende-se que a escolha é válida, pois os artigos relacionados a esses meios de comunicação geralmente antecedem livros consagrados, são considerados fontes seguras para pesquisas e devem apresentar rigor metodológico para publicação. No entanto, cabe destacar que as bases apresentam classificações divergentes em alguns casos. Sendo assim, os dados coletados na ISI (2009) e na Scopus (2009) foram submetidos a processos específicos de tratamento e análise de consistência, conforme detalhamento apresentado no próximo tópico.

\subsection{Tratamento dos Dados}

Tendo em vista que as bases de dados escolhidas para esta pesquisa apresentam, por exemplo, diferenças de nomenclaturas das fontes de publicações e os nomes dos autores grafados de maneira distinta, adotou-se um conjunto de processos independentes para o tratamento dos dados, como mostra a Figura 2. 


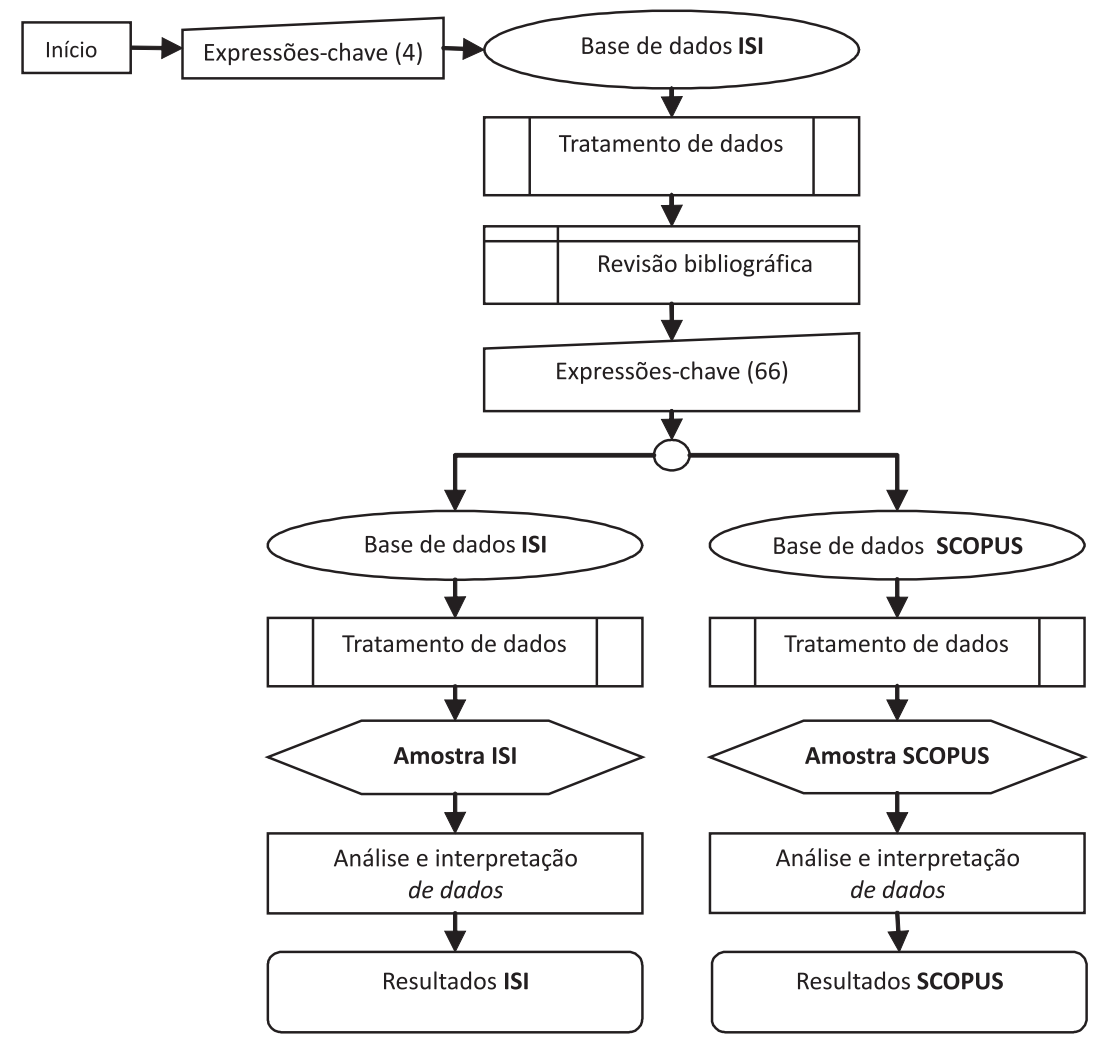

Figura 2: Fluxograma do processo de amostragem e análise dos dados Fonte: Elaborada pelos autores

Uma vez coletados os 3.688 registros na base ISI (2009), a partir de buscas feitas com as 66 expressões identificadas na revisão bibliográfica, algumas intervenções no banco se fizeram necessárias. A primeira delas foi eliminar fichas de artigos que não estavam completas. Notou-se que alguns artigos não tinham indicados, por exemplo, os autores dos trabalhos, o que redundou na eliminação de 131 elementos. A segunda ação foi a padronização dos nomes de autores e fontes de publicação. Neste caso, uma análise de consistência detalhada no banco foi empreendida.

Com os dados padronizados foi possível se detectar, então, as duplicidades. Por se tratar de "expressões-sinônimo ou correlatas", alguns artigos foram incluídos na base em mais de um termo-chave. A busca por registros repetidos resultou na retirada de 584 elementos do conjunto. Julgase oportuno comunicar que 17 desses apresentavam fontes de publicação distintas, embora todos os outros dados fossem coincidentes. Então, decidiu- 
se pela aplicação de três critérios para a manutenção ou eliminação de dados do banco, nesta ordem: (1) as publicações mais antigas foram mantidas, (2) o registro com maior número de citações foi preservado, caso o ano de publicação fosse o mesmo e, (3) em se tratando do mesmo ano de publicação e do mesmo número de citações, arbitrariamente eliminou-se o(s) registro(s) sobressalente(s).

Por fim, foram expurgados do banco de dados os registros que não diziam respeito às fontes de publicação periódicos e congressos (13 patentes), além daqueles que não estavam compreendidos entre 1989 e 2008 . A amostra ISI, portanto, foi composta por 2.747 artigos.

O mesmo processo, então, repetiu-se na base de dados Scopus (2009). Inicialmente, foram levantadas 8.604 fichas de artigos. Destas, 1.243 foram retiradas do conjunto por estarem incompletas e 3.799 foram apagadas, uma vez que continham dados repetidos. Conforme verificou-se no tratamento dos dados da primeira base, diferentes expressões podem conduzir os pesquisadores aos mesmos artigos.

A base Scopus (2009), diferentemente da ISI (2009), gera como resultados de busca, além dos artigos e das patentes, outros tipos de publicações. Entre livros, notas, relatórios, artigos de negócios e de imprensa, erratas, short surveys e reviews, foram encontrados 585 registros. Somou-se a esses mais de 160 trabalhos publicados fora do período de observação desta pesquisa $e$ todos foram devidamente retirados do conjunto de dados. Constituiu-se, portanto, uma amostra Scopus com 2.813 elementos. A próxima seção mostra o que resultou da análise dos dados.

\section{Análise dos Resultados}

A análise e interpretação dos dados deram-se em três frentes, baseadas nas leis e princípios bibliométricos. A seguir encontra-se síntese de cada uma delas.

a) Lei de Zipf: expressões-chave mais usadas por pesquisadores (classificação por número de artigos identificados).

b) Lei de Lotka e análise de citação: número de citações por autor (classificação dos autores mais citados), número de artigos publica- 
dos por autor e número de citações por artigo (classificação dos artigos mais citados).

c) Lei de Bradford: número de citações por periódico (classificação por periódicos mais consultados) e número de artigos publicados pelos principais periódicos.

Na seção intitulada Metodologia, foram identificadas 66 expressões que direcionam interessados no tema alianças estratégicas ao conteúdo publicado nas últimas duas décadas. Porém, embora todas possibilitem o acesso a alguma fração desse material, não apresentam o mesmo grau de ocorrência ou probabilidade de sucesso em uma busca. O Gráfico 1 indica aquelas responsáveis pelo levantamento de $80 \%$ dos registros componentes das amostras deste trabalho.

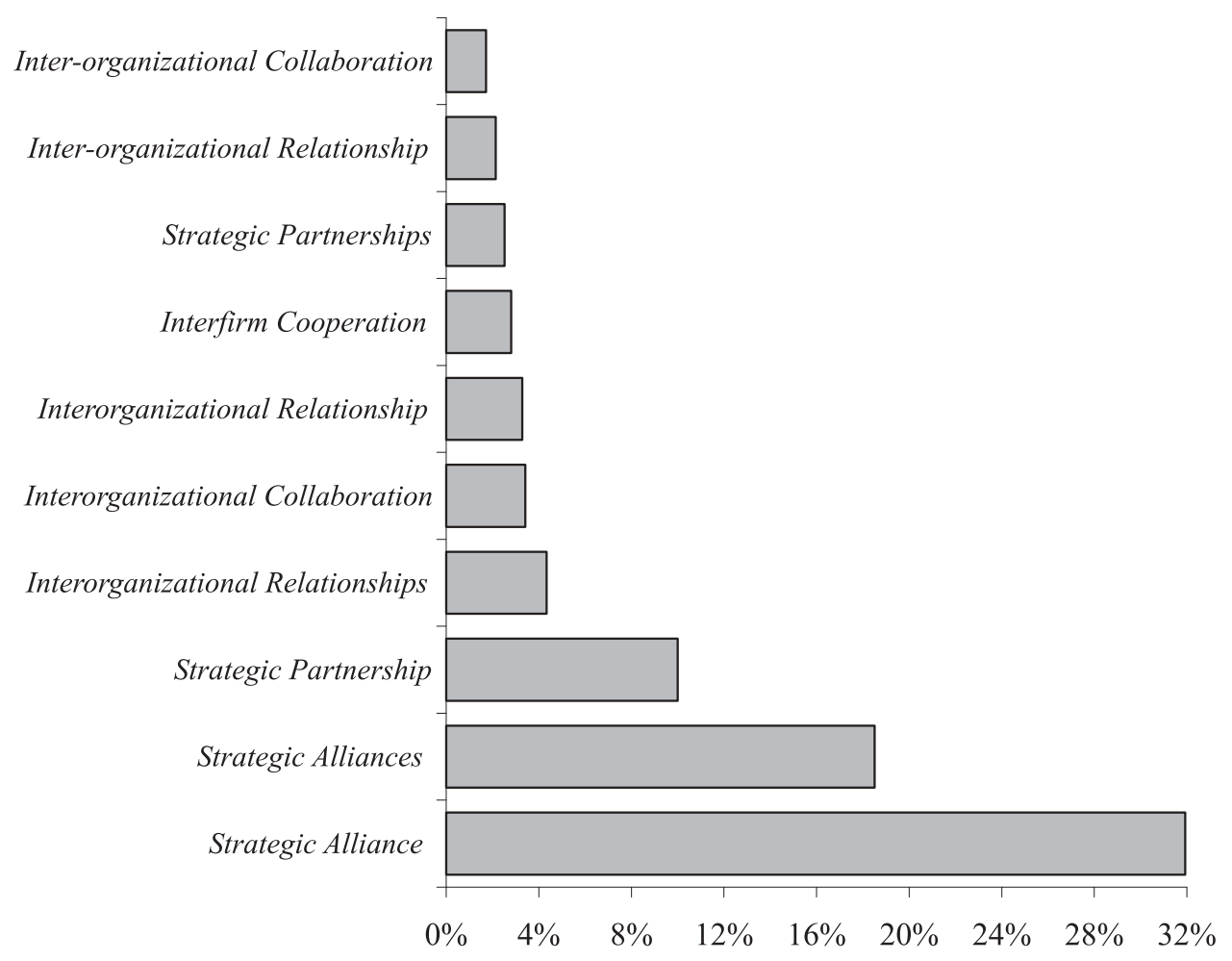

Gráfico 1: Expressões-chave mais usadas por pesquisadores Fonte: Elaborado pelos autores

Nota-se a predominância de três das quatro expressões-chave usadas no início do processo amostral. Os termos strategic alliance, strategic alliances 
e strategic partnership respondem por mais $60 \%$ dos dados disponíveis nas bases ISI (2009) e Scopus (2009).

Uma vez atendida a quarta parte dos objetivos deste estudo, faz-se necessário responder aos outros $3 / 4$ da questão de pesquisa: quais são os autores, artigos e periódicos mais relevantes sobre o tema alianças estratégi-

\section{cas entre organizações?}

Segue, portanto, a Tabela 2, que indica os autores mais referenciados na produção científica analisada. Os campos sombreados mostram a intersecção existente entre as amostras construídas.

Tabela 2: Autores mais citados (20 primeiros)

\begin{tabular}{|l|r|r|}
\hline \multicolumn{3}{|c|}{ ISI WEB OF KNOWLEDGE } \\
\hline & $\begin{array}{c}\text { Número de } \\
\text { Citações }\end{array}$ & $\begin{array}{c}\text { Número de } \\
\text { Artigos }\end{array}$ \\
\hline R. Gulati & 2.150 & 11 \\
\hline H. Singh & 1.381 & 6 \\
\hline J. Hagedoorn & 1.185 & 26 \\
\hline W. W. Powell & 1.154 & 5 \\
\hline J. H. Dyer & 1.040 & 2 \\
\hline K. W. Koput & 947 & 2 \\
\hline A. Parkhe & 943 & 8 \\
\hline L. Smith-Doerr & 844 & 1 \\
\hline K. M. Eisenhardt & 711 & 3 \\
\hline G. Hamel & 706 & 1 \\
\hline J. E. Oxley & 700 & 5 \\
\hline A. C. Inkpen & 690 & 9 \\
\hline B. S. Teng & 686 & 9 \\
\hline T. K. Das & 684 & 10 \\
\hline P. S. Ring & 682 & 4 \\
\hline B. S. Silverman & 679 & 5 \\
\hline T. E. Stuart & 673 & 7 \\
\hline A. H. Vandeven & 591 & 1 \\
\hline D. C. Mowery & 467 & 2 \\
\hline B. L. Simonin & 437 & 4 \\
\hline E. & & \\
\hline
\end{tabular}

\begin{tabular}{|l|r|r|}
\hline \multicolumn{3}{|c|}{ SCOPUS } \\
\hline AUTOR & $\begin{array}{c}\text { Número de } \\
\text { Citações }\end{array}$ & $\begin{array}{c}\text { Número de } \\
\text { Artigos }\end{array}$ \\
\hline W. W. Powell & 1.274 & 3 \\
\hline R. Gulati & 1.261 & 6 \\
\hline K. W. Koput & 1.193 & 2 \\
\hline T. K. Das & 1.177 & 17 \\
\hline B. S. Teng & 1.158 & 14 \\
\hline L. Smith-Doerr & 1.064 & 1 \\
\hline H. Singh & 889 & 7 \\
\hline K. M. Eisenhardt & 885 & 2 \\
\hline B. S. Silverman & 788 & 4 \\
\hline J. E. Oxley & 767 & 4 \\
\hline T. E. Stuart & 754 & 6 \\
\hline J. Hagedoorn & 618 & 13 \\
\hline B. L. Simonin & 550 & 4 \\
\hline D. C. Mowery & 546 & 2 \\
\hline S.L. Brown & 530 & 1 \\
\hline G. Ahuja & 489 & 2 \\
\hline Y.L. Doz & 486 & 2 \\
\hline P. Kale & 405 & 3 \\
\hline J. H. Dyer & 394 & 3 \\
\hline C.B. Schoonhoven & 355 & 1 \\
\hline S. & & \\
\hline
\end{tabular}

Fonte: Adaptada de ISI (2009) e Scopus (2009)

Embora uma análise superficial do resultado acima ofereça a resposta desejada para este estudo, cabe aprofundar um pouco mais a investigação com o intuito de se descobrir a origem dos pesquisadores citados, e, portanto, os núcleos de pesquisa aos quais estão filiados.

Boston University, Carlson School of Management, City University of New York, George Washington University, Harvard Business School, Hass 
School of Business, International Business Kelley School, Jesse H. Jones Graduate School of Management, Loyola Marymount University, Marriott School, Stanford University, The Fetcher School, The Paul Merage School of Business, Thunderbird School of Global Management, University of Chicago e Wharton School, entre outras escolas norte americanas, despontam como centros de excelência em pesquisas sobre alianças estratégicas.

Além dos Estados Unidos, os países que sediam núcleos também desenvolvidos são Holanda, Canadá, Inglaterra e França, respectivamente com destaques para a Maastricht University, Rotman School, London Business School e Insead.

A Tabela 3, então, relaciona os artigos mais citados por pesquisadores de todo o mundo.

Tabela 3: Artigos mais citados (20 primeiros)

\begin{tabular}{|l|r|}
\hline \multicolumn{2}{|c|}{ ISI WEB OF KNOWLEDGE } \\
\hline \multicolumn{1}{|c|}{ ARTIGO } & $\begin{array}{c}\text { Número de } \\
\text { Citações }\end{array}$ \\
\hline Powell; Koput; Smith-Doerr, 1996 & 844 \\
\hline Dyer; Singh, 1998 & 811 \\
\hline Hamel, 1991 & 706 \\
\hline Gulati, 1995 & 657 \\
\hline Ring; Vandeven, 1994 & 591 \\
\hline Gulati, 1998 & 479 \\
\hline Brown; Eisenhardt, 1997 & 430 \\
\hline Parkhe, 1993 & 417 \\
\hline Webster, 1992 & 394 \\
\hline Mowery; Oxley; Silverman, 1996 & 377 \\
\hline Mohr; Spekman, 1994 & 375 \\
\hline Hagedoorn, 1993 & 356 \\
\hline Oliver, 1990 & 347 \\
\hline Doz, 1996 & 331 \\
\hline Das; Teng, 1998 & 322 \\
\hline Gulati, 1995 & 312 \\
\hline Ahuja, 2000 & 278 \\
\hline Eisenhardt; Schoonhoven, 1996 & 276 \\
\hline Inkpen; Beamish, 1997 & 267 \\
\hline Gulati, 1999 & 249 \\
\hline
\end{tabular}

\begin{tabular}{|c|c|}
\hline \multicolumn{2}{|l|}{ SCOPUS } \\
\hline ARTIGO & $\begin{array}{c}\text { Número de } \\
\text { Citações }\end{array}$ \\
\hline Powell; Koput; Smith-Doerr, 1996 & 1.064 \\
\hline Gulati, 1998 & 576 \\
\hline Brown; Eisenhardt, 1997 & 530 \\
\hline Mowery; Oxley; Silverman, 1996 & 433 \\
\hline Doz, 1996 & 423 \\
\hline Das; Teng, 1998 & 418 \\
\hline Eisenhardt; Schoonhoven, 1996 & 355 \\
\hline Ahuja, 2000 & 318 \\
\hline Kale; Singh; Perlmutter, 2000 & 303 \\
\hline Gulati, 1999 & 297 \\
\hline Dyer, 1997 & 292 \\
\hline Stuart; Hoang; Hybels, 1999 & 280 \\
\hline Teece, 1992 & 257 \\
\hline Gulati; Singh, 1998 & 256 \\
\hline Simonin, 1999 & 250 \\
\hline Baum; Calabrese; Silverman, 2000 & 230 \\
\hline Anand; Khanna, 2000 & 221 \\
\hline Phillips; Shane; Oviatt, 1994 & 220 \\
\hline Hart; Saunders, 1997 & 204 \\
\hline Das; Teng, 2000 & 201 \\
\hline
\end{tabular}

Fonte: Adaptada de ISI (2009) e Scopus (2009)

A Tabela 4, por fim, aponta os periódicos que deram mais destaque ao tema deste trabalho nos últimos 20 anos. Assim como ocorre com os núcleos 
de pesquisa, os periódicos mais bem-sucedidos na publicação de artigos sobre alianças estratégicas estão sediados nos Estados Unidos.

Tabela 4: Periódicos mais citados (20 primeiros)

\begin{tabular}{|c|c|c|c|c|c|}
\hline \multicolumn{3}{|c|}{ ISI WEB OF KNOWLEDGE } & \multicolumn{3}{|l|}{ SCOPUS } \\
\hline PERIÓDICO & $\begin{array}{l}\text { Número de } \\
\text { Citações }\end{array}$ & $\begin{array}{c}\text { Número de } \\
\text { Artigos }\end{array}$ & PERIÓDICO & $\begin{array}{l}\text { Número de } \\
\text { Citações }\end{array}$ & $\begin{array}{c}\text { Número de } \\
\text { Artigos }\end{array}$ \\
\hline Strategic Management Journal & 7.128 & 102 & Strategic Management Journal & 5.502 & 55 \\
\hline Academy of Management Journal & 3.459 & 45 & Administrative Science Quarterly & 2.737 & 11 \\
\hline Academy of Management Review & 3.335 & 27 & Organization Science & 1.874 & 27 \\
\hline Administrative Science Quarterly & 2.844 & 21 & Journal of Business Venturing & 929 & 21 \\
\hline Organization Science & 2.554 & 52 & Academy of Management Journal & 915 & 12 \\
\hline Journal of Intl. Business Studies & 1.591 & 48 & Academy of Management Review & 817 & 5 \\
\hline Research Policy & 1.044 & 58 & Research Policy & 789 & 35 \\
\hline Journal of Business Venturing & 786 & 29 & \begin{tabular}{|l|} 
Journal of Management \\
\end{tabular} & 646 & 9 \\
\hline \begin{tabular}{|l|} 
Journal of Management Studies \\
\end{tabular} & 652 & 53 & Journal of Intl. Business Studies & 597 & 13 \\
\hline Organization Studies & 629 & 31 & Organization Studies & 586 & 18 \\
\hline Journal of Marketing & 618 & 13 & \begin{tabular}{|l|} 
Journal of Management Studies \\
\end{tabular} & 526 & 19 \\
\hline Journal of Management & 541 & 19 & Journal of Economic Bhv. \& Org. & 418 & 8 \\
\hline Management Science & 427 & 15 & Academy of Management Executive & 387 & 10 \\
\hline Journal of Economic Bhv. \& Org. & 419 & 13 & Long Range Planning & 384 & 26 \\
\hline \begin{tabular}{|l} 
Journal of World Business \\
\end{tabular} & 350 & 22 & Journal of Business Research & 332 & 35 \\
\hline Long Range Planning & 345 & 36 & European Management Journal & 300 & 30 \\
\hline Technovation & 342 & 45 & Industrial Marketing Management & 269 & 27 \\
\hline Journal of Business Research & 331 & 43 & Information And Management & 240 & 15 \\
\hline Regional Studies & 317 & 15 & Management Science & 231 & 11 \\
\hline Mis Quarterly & 313 & 8 & Mis Quarterly & 228 & 6 \\
\hline
\end{tabular}

Fonte: Adaptada de ISI (2009) e Scopus (2009)

Ainda que os resultados apresentados sejam considerados satisfatórios, há limitações intrínsecas a esta pesquisa que devem ser apontadas, sobretudo com relação à grande concentração da produção em poucos países. $\mathrm{Na}$ próxima seção, tais limitações são discutidas, além de sugestões para futuros trabalhos e as considerações finais.

\section{Considerações Finais}

A identificação e organização das expressões-chave de busca, dos principais autores, artigos e periódicos sobre alianças estratégicas - objetivos enunciados no tópico introdutório deste estudo - foram alcançados, sendo as primeiras listadas na Seção 4 e os demais apresentados na Análise dos Resultados.

Cabe mencionar que as realizações descritas acima contribuem sobremaneira para a consolidação do conhecimento disponível, para o desenvol- 
vimento de novas visões e conceitos, além de significarem um ponto de partida para estudiosos encontrarem respostas que ainda não tenham sido dadas no referido campo de pesquisa.

Faz-se necessário, porém, mostrar algumas limitações do método e das fontes de dados que estão disponíveis para a construção de trabalhos dessa natureza nos dias de hoje. As bases de dados escolhidas para esta investigação - ISI (2009) e Scopus (2009) - não abrangem a produção acadêmica de muitos países, sobretudo aqueles em desenvolvimento, e tem a predominância de publicações norte-americanas e em língua inglesa. Sendo assim, publicações brasileiras, relevantes para o entendimento do problema de pesquisa no país e outros relacionados, não necessariamente integram as amostras utilizadas, e por consequência, não estão refletidas nos resultados.

Outra limitação, originada também no processo amostral, diz respeito ao tipo de publicações ou meios de comunicação selecionados. Livros influentes sobre alianças estratégicas, por exemplo, não são muito comuns nas fontes de dados. Ou seja, o conhecimento que não tenha sido transformado em um artigo científico não foi levado em consideração nesta pesquisa.

O que se sugere, então, é o desenvolvimento de uma investigação, cuja amostra seja constituída por artigos publicados no Brasil, ainda que não se considere outros veículos de publicação, mas que seja possível a identificação de autores e núcleos de pesquisa com produção relevante no país. Conforme enunciado em Goffman e Newill (1967), citados por Araujo (2006), a dinâmica da transmissão do conhecimento passa pelas redes sociais, portanto é fundamental que sejam identificados e fortalecidos os centros de pesquisa.

Um bom exemplo disso foi dado pela Wharton School e o Insead, referências no desenvolvimento de estudos em alianças estratégicas, ao se associarem para formar uma rede global de negócios em educação e conhecimento - global business education and knowledge network. Quanto melhores forem as condições internas para o desenvolvimento de projetos e externas para propagação da informação, maior será a participação de cada núcleo na geração de conhecimento.

Por fim, sugere-se o emprego da análise de cocitação em futuros trabalhos com o intuito de identificar grupos de estudiosos e interações entre pesquisadores. Uma análise desse tipo pode apontar, inclusive, prováveis linhas de pesquisas existentes no campo das alianças entre organizações. 


\section{Strategic Alliances: a bibliometric analysis of scientific output between 1989 and 2008}

\section{Abstract}

Organizing data from scientific outputs has been a powerful toll as it contributes to the continuity of research and development of a specific knowledge theme. The main purpose of this study, therefore, is to quantitatively analyze what occurred in the field of strategic alliances between 1989 and 2008. Relying on bibliometric techniques and literature, it has been found 66 key-words driving researchers to a variety of content about this topic. Moreover, two samples of articles from ISI Web of Knowledge and Scopus databases were built up with, respectively, 2.747 and 2.813 items. These samples allowed the identification of the most referred authors, articles and periodicals by scholars around the world. This research then becomes an important ally to the development of new ideas, concepts and prospects for approaches to strategic alliances.

Key words: Strategic alliance. Bibliometric analysis. Strategic partnership.

\section{Referências}

AHUJA, G. Collaboration networks, structural holes, and innovation: a longitudinal study. Administrative Science Quarterly, v. 45, p. 425-455, sep. 2000.

ANAND, Bharat N.; KHANNA, Tarun. Do firms learn to creat value? The case of alliances. Strategic Management Journal, v. 21, p. 295-315, mar. 2000.

ARAUJO, Carlos. Bibliometria: evolução histórica e questões atuais. Pesquisa Brasileira em Ciência da Informação e Biblioteconomia, v. 2, p.11-32, jan-jun. 2006.

ARCHAMBAULT, Eric; CAMPBELL, David; GINGRAS, Yves; LARIVIERE, Vincent. Comparing bibliometric statistics obtained from the Web of Science and Scopus. Journal of American Society for Information science and Technology, v. 60, p. 1320-1326, jul. 2009.

BARROS, Betânia Tanure de. Fusões, aquisições \& parcerias. São Paulo: Atlas, 2001. 185p. 
BAUM, J. A. C.; CALABRESE, T.; SILVERMAN, B. S. Don't go it alone: alliance network composition and startups' performance in Canadian biotechnology.

Strategic Management Journal, v. 21, p. 267-294, mar. 2000.

BROWN, S. L.; EISENHARDT, K. M. The art of continuous change: Linking complexity theory and time-paced evolution in relentlessly shifting organizations. Administrative Science Quarterly, v. 42, p. 1-34, mar. 1997.

DAS, T. K.; TENG, B. S. Between trust and control: developing confidence in partner cooperation in alliances. Academy of Management Review, v. 23, p. 491-512, jul. 1998.

. A resource-based theory of strategic alliances. Journal of Management, v. 2, p. 31-61, jan. 2000.

DOZ, Y. L. The evolution of cooperation in strategic alliances: Initial conditions or learning processes? Strategic Management Journal, v. 17, p. 55-83, summer 1996.

DYER, J. H. Effective interfirm collaboration: how firms minimize transaction costs and maximize transaction value. Strategic Management Journal, v. 18 , p. 535-556, aug. 1997.

DYER, J. H.; SINGH, H. The relational view: cooperative strategy and sources of interorganizational competitive advantage. Academy of Management Review, v. 23, p. 660-679, oct. 1998.

EISENHARDT, K. M.; SCHOONHOVEN, C. B. Resource-based view of strategic alliance formation: Strategic and social effects in entrepreneurial firms. Organization Science, v. 7, p. 136-150, mar-apr. 1996.

FERREIRA, Aurélio Buarque de Holanda. Novo Aurélio século XXI: o dicionário da língua portuguesa. Rio de Janeiro: Nova Fronteira, 1999.

GUEDES, Vania; BORSCHIVER, Suzana. Bibliometria: uma ferramenta estatística para a gestão da informação e do conhecimento, em sistemas de informação, de comunicação e de avaliação científica e tecnológica. CINFORM Encontro Nacional de Ciência da Informação VI, v. 6, dez. 2005.

GULATI, R. Does familiarity breed trust: the implications of repeated ties for contractual choice in alliances. Academy of Management Journal, v. 38, p. 85-112, feb. 1995. 
. Social structure and alliance formation patterns: a longitudinal analysis. Administrative Science Quarterly, v. 40, p. 619-652, dec. 1995.

. Alliances and networks. Strategic Management Journal, v. 19, p. 293-317, apr. 1998.

. Network location and learning: the influence of network resources and firm capabilities on alliance formation. Strategic Management Journal, v. 20, p. 397-420, may, 1999.

GULATI, R.; SINGH, H. The architecture of cooperation: managing coordination costs and appropriation concerns in strategic alliances. Administrative Science Quartely, v. 43, p. 781-814, dec. 1998.

HAGEDOORN, J. Understanding the rationale of strategic technology partnering: interorganizational modes of cooperation and sectoral differences. Strategic Management Journal, v. 14, p. 371-385, jul. 1993.

HAMEL, G. Competition for competence and inter-partner learning with international strategic alliances. Strategic Management Journal, v. 12, p. 83-103, summer 1991.

HART, P.; SAUNDERS, C. Power and trust: critical factors in the adoption and use of electronic data interchange. Organization Science, v. 8, p. 23-42, janfev. 1997.

INKPEN, A. C.; BEAMISH, P. W. Knowledge, bargaining power, and the instability of international joint ventures. Academy of Management Review, v. 22, p. 177-202, jan. 1997.

ISI - ISI WEB OF KNOWLEDGE. Homepage. Disponível em: < http:// isiwebofknowledge.com/>. Acesso em: $1^{\circ}$ jul. 2009.

KALE, P.; SINGH, H.; PERLMUTTER, H. Learning and protection of proprietary assets in strategic alliances: Building relational capital. Strategic Management Journal, v. 21, p. 217-237, mar. 2000.

LORANGE, Peter; ROOS, Johan. Strategic alliances: formation, implementation and evolution. Malden: Blackwell, 1993. 295p.

MARTINS, Gilberto de Andrade; THEÓPHILO, Carlos Renato. Metodologia da investigação científica para ciências sociais aplicadas. São Paulo: Atlas, 2007. 
MOHR, J.; SPEKMAN, R. Characteristics of partnership success: partnership attributes, communication behavior, and conflict-resolution techniques.

Strategic Management Journal, v. 15, p. 135-152, fev. 1994.

MOWERY, D. C.; OXLEY, J. E.; SILVERMAN, B. S. Strategic alliances and interfirm knowledge transfer. Strategic Management Journal, v. 17, p. 77-91, winter 1996.

OLIVER, C. Determinants of interorganizational relationships: integration and future-directions. Academy of Management Review, v. 15, p. 241-265, 1990.

PARKHE, A. Strategic alliance structuring: a game-theoretic and transaction cost examination of interfirm cooperation. Academy of Management Journal, v. 36, p. 794-829, aug. 1993.

PILKINGTON, Alan; MEREDITH, Jack. The evolution of the intellectual structure of operations management - 1980-2006: a citation/co-citation analysis. Journal of Operations Management, v. 27, p. 185-202, jun. 2009.

PHILLIPS, McDougall P.; SHANE, S.; OVIATT, B. M. Explaining the formation of international new ventures: The limits of theories from international business research. Journal of Business Venturing, v. 9, p. 469-487, nov. 1994.

POWELL, W. W.; KOPUT, K. W.; SMITH-DOERR, L. Interorganizational collaboration and the locus of innovation: networks of learning in biotechnology. Administrative Science Quarterly, v. 41, p. 116 -145, mar. 1996.

RAMOS-RODRIGUEZ, Antônio; RUIZ-NAVARRO, José. Changes in the intellectual structure of strategic management research: a bibliometric study of the strategic management journal, 1980-2000. Strategic Management Journal, v. 25, p. 981-1004, aug. 2004.

RIBEIRO, Áurea Helena Puga; SILVA JUNIOR, Antônio Batista da. Parcerias e alianças estratégicas. Fusões, Aquisições \& Parcerias. p. 89-101, 2001.

RING, P. S.; VANDEVEN, A. H. Developmental processes of cooperative interorganizational relationships. Academy of Management Review, v. 19, p. 90-118, jan. 1994.

ROBINSON, D. T. Strategic alliances and the boundaries of the firm. Review of Financial Studies, v. 21, p. 649-681, summer, 2000. 
SCOPUS - Scopus Citation Tracker. Homepage. Disponível em: <http:// www.scopus.com/home.url >. Acesso em: $1^{\circ}$ jul. 2009.

SEM, S. For what purpose are bibliometric indicators and how should they work? University of Calcutta, India.1999.

SIMONIN, B. L. Ambiguity and the process of knowledge transfer in strategic alliances. Strategic Management Journal, v. 20, p. 595-623, jul. 1999.

STUART, T. E.; HOANG, H.; HYBELS, R. C. Interorganizational endorsements and the performance of entrepreneurial ventures. Administrative Science Quarterly, v. 44, p. 315-349, jun. 1999.

TEECE, D.J. Competition, cooperation, and innovation. organizational arrangements for regimes of rapid technological progress. Journal of Economic Behavior and Organization, v. 18, p. 1-25, jun. 1992.

WEBSTER, F. E. The changing-role of marketing in the corporation. Journal of Marketing, v. 56, p. 1-17, oct. 1992.

YOSHINO, Michael Y; RAGAN, U. Srinivasa. Strategic alliances: an entrepreneurial approach to globalization. Boston: Harvard Business School Press, 1995. 259p. 